\title{
The Devil's Party: Milton en la poética de William Blake
}

\author{
Adrián MUÑOZ \\ Universidad Nacional Autónoma de México
}

\begin{abstract}
La imaginería de William Blake está fuertemente anclada en el simbolismo religioso en torno de la Biblia. Con todo, dicha imaginería está filtrada por la particular ideología de Blake, para quien el poeta es una manifestación legítima y óptima del profeta bíblico. Para Blake, John Milton constituye uno de los paradigmas de la figura del poeta-profeta en lengua inglesa. Este ensayo se propone explorar los modos en que Blake incorporó la obra y la figura de Milton en su proyecto poético, tanto a través de los ecos de la obra miltoniana como de su interpretación de la ideología de Milton. La discusión se centra particularmente en el poema Milton a Poem in Two Books y analiza las tensiones mentales y estéticas de Blake en dicha obra.
\end{abstract}

Palabras Clave: William Blake, Milton a Poem in Two Books, romanticismo inglés, John Milton-influencia en la literatura inglesa, literatura y religión.

William Blake's imagery is strongly rooted in a religious symbolism that springs from Biblical lore. Yet this imagery is determined by Blake's own ideology, according to which the poet is a most legitimate incarnation of the biblical prophets. For Blake, John Milton stands out as one of the main models of the poet-prophet within the English literary tradition. This paper explores the ways in which Blake incorporated the work and figure of Milton in his own poetic project, both through the echoes of the Miltonic work and Blake's interpretation of Milton's ideology. The paper especially focuses on Milton, a Poem in Two Books and analyses both the mental and aesthetic tensions of Blake within the poem.

KeY Words: William Blake, Milton a Poem in Two Book, english romanticism, John Milton-influence on English literature, literature and religion.

Si bien es cierto que la figura de John Milton no es omnipresente en la obra de William Blake, la influencia miltoniana sí es significativamente recurrente. Esta influencia, sin embargo, trasciende el ámbito literario. Lo que Blake toma de Milton no es únicamente un talento literario que sirva de modelo para algunos de sus propios trabajos poéticos, sino una motivación emotiva que trasciende la esfera meramente estética.

\footnotetext{
${ }^{1}$ Este artículo está basado en una ponencia presentada durante el Coloquio 2008, Año Miltoniano (FFL-UNAM, 30 de octubre de 2008).
} 


\section{$64 \square$ THE DEVIL'S PARTY: MILTON EN LA POÉTICA}

Milton es importante para Blake no sólo porque es un gran escritor, sino porque, a ojos de Blake, Milton pertenece a la misma categoría de Chaucer, Shakespeare, Isaías, Ezequiel, etcétera, es decir, la de poetas-profetas, denominados por Blake los Hijos de Los, el Eterno Profeta. Salvo por Samson Agonistes, Blake se dedicó a ilustrar las obras poéticas principales de John Milton y, como es de esperar, Paradise Lost representa una obra paradigmática y de especial relevancia para Blake. Lo que Blake encuentra en El paraíso perdido o Paradise Lost no es sólo un impresionante poema épico, escrito con pulcritud y perfección, sino un depósito de imaginación agolpada. Se trata del arte de la versificación al servicio del arte visionario.

Foster Damon traza varias semejanzas entre la vida de Milton y la de Blake que resultan notorias. Pero acaso las semejanzas más interesantes son las que se refieren al amplio proyecto poético de cada uno de ellos (Damon, 1988: 274-275). Si John Milton ideó L'Allegro e Il Penseroso como una suerte de poema circular que de algún modo no concluye nunca, Blake conformó sus Songs of Innocence y Songs of Experience de un modo similar. En los Cantos también encontramos una serie de correspondencias que se complementan sistemáticamente y no sólo al azar. The Book of Thel de Blake corresponde a Comus de Milton. En ambos textos, una joven doncella tiene que lidiar con la posibilidad de la experiencia sensual, si bien los métodos, las tensiones y las voces empleadas difieren en las dos obras. Según Damon, Visions of the Daughters of Albion responde a los panfletos que Milton escribió sobre el divorcio, aunque Blake se preocupó más por defender la libertad sexual absoluta y no únicamente la disolución del vínculo matrimonial. Si por un lado la faceta política de Milton lo llevó a escribir obras en contra de ciertos sistemas políticos, Blake, a través de obras como America, enaltece el papel de la revolución.

La obra cúspide de Milton, Paradise Lost (1667/1674), encuentra su paralelo en The Four Zoas. Las dos obras comparten ciertos artificios narrativos y abordan el mismo tema: la salvación de la humanidad. Por supuesto, The Four Zoas es mucho más que un mero calco del gran poema de Milton. John Milton se ciñe a un imaginario cristiano, mientras que Blake combina elementos cristianos con paganos y con figuras surgidas de su propia cosmovisión.

El caso de The Marriage of Heaven and Hell (MHH) es interesante porque a pesar de que la presencia de Milton es evidente, Blake parece no coincidir del todo con su antecesor. The Marriage podría corresponder a De doctrina Christiana de Milton, sólo que este último consolida sistemas de pensamiento religioso más o menos convencionales, mientras que la obra de Blake los derriba con objeto de construir uno nuevo, uno donde los papeles del deseo y la imaginación obtengan prominencia. ${ }^{2}$ Para ello Blake, vía la Voz del Diablo, sostiene que los personajes de la obra miltoniana tienen los nombres equivocados: lo que Milton presenta como el Mesías es en realidad Satanás y viceversa, y ello se refleja en los valores de la represión y la libertad del deseo:

\footnotetext{
${ }^{2}$ Hay que advertir, sin embargo, que De doctrina Christiana no fue publicado sino hasta 1823-1825, es decir, después de que Blake ya había completado la mayoría de sus obras.
} 
Those who restrain desire do so because theirs is weak enough to be restrained; and the restrainer or reason usurps its place $\&$ governs the unwilling.

And being restrain'd, it by degrees becomes passive, till it is only the shadow of desire.

The history of this is written in Paradise Lost, \& the Governor or Reason is call'd Messiah.

And the original Archangel, or possessor of the command of the heavenly host, is call'd the Devil or Satan, and his children are call'd Sin \& Death (MHH, pl. 5).

Este pasaje, como han advertido ya otros estudiosos antes, remite a las diversas actividades de las hordas infernales y al paso de Satanás por el Caos según se describe en el Libro II de Paradise Lost (cf. Tannenbaum, 1976: 78). Uno de los rasgos más significativos de MHH es que Blake interpreta al Satán de Paradise Lost de manera que se convierte en el estandarte del refinamiento estético y en la voz que transmite los "principios del deseo" (Schock, 1993: 459). La interpretación que Blake hace de la obra y la persona de John Milton no se ciñe sólo a The Marriage of Heaven and Hell.

Significativamente, Blake compuso también un largo poema narrativo intitulado Milton a Poem in Two Books (circa 1804-1810), donde el poeta explora la evolución espiritual del autor de Paradise Lost. El poema Milton (Mil) fue concebido e ilustrado hacia 1804, más de diez años después de The Marriage of Heaven and Hell y más de cien años tras la muerte de John Milton. En un sentido general, el texto aborda el regreso a la tierra de Milton, quien, en su encuentro con William Blake, también personaje de este poema, reflexiona sobre el carácter de la creación poética. El viaje místico que emprenden ambos los lleva a analizar los aciertos y yerros de sus predecesores y contemporáneos. De manera importante, Blake retoma un tópico que ya había introducido en MHH, a saber: los errores poético-visionarios de John Milton. En palabras de Leslie Tannenbaum, lo que Blake hace es una "lectura correctiva de Milton" (Tannenbaum, 1976: 78). Como veremos, sin embargo, el mundo mitológico de Milton a Poem -y por ende la comprensión del mismo - es harto compleja, pues está imbuida del imaginario mitológico del autor.

La portada de Milton incluye un epígrafe en la parte inferior que reza: "To justify the Ways of God to Men", que corresponde con el verso número veintiséis de Paradise Lost:

I may assert Eternal Providence,

And justify the ways of God to men (ParL 1.25-26).

Así, Blake pone de manifiesto su deuda para con la obra de Milton; pone también de relieve el hecho de que ambas composiciones poseen el mismo objetivo. Como bien puntualiza Bloom (1999: 197), los dos grandes poemas, el de Milton y el de Blake, comparten con el Libro de Job el objetivo de presentar una teodicea, es decir, abordar el problema del mal y, por tanto, "justificar los caminos de Dios". Por supuesto, la teo- 
dicea blakeana no es idéntica a la visión miltoniana: "But in the Book of Job, Milton's Messiah is call'd Satan" (MHH, pl. 5).

Aunque en Milton a Poem Blake no elaborará directamente sobre las tipologías desarrolladas en The Marriage, la misma idea permanece, a saber: que los nombres otorgados por Milton a sus personajes en Paradise Lost estaban equivocados, y que quienes ostentaban los títulos honoríficos eran en realidad agentes del mal.

Los errores que John Milton tendrá que corregir en el poema homónimo de Blake se resumen de este modo: "The reason Milton wrote in fetters when he wrote of Angels \& God, and at liberty when of Devils and Hell, is because he was a true Poet and of the Devil's party without knowing it" (MHH, pl. 5).

Evidentemente, cuando Blake habla aquí del "bando del Diablo" (Devil's party), se refiere en realidad a los espíritus libres, en oposición a los tiránicos seres de la razón y la moral que se adjudican el título de "Buenos". Particularmente, Blake consideraba que el principal error de Milton había sido presentar a un Dios cruel, distante y frío en Paradise Lost. De manera implícita, Blake habría también de contrarrestar el esquema arriano de Milton, esquema que niega el concepto de la Trinidad cristiana, colocando en la cúspide a Dios Padre/Yahvé y privando a Dios Hijo/Jesús de una naturaleza verdaderamente divina ( $c f$. Nuttall, 2007: 136-140 y 164-165). Para Blake, la salvación individual debe llevar a la ascensión de Albión, el Hombre Universal, y a la comunión con Cristo. Estamos, pues, ante una confrontación ideológica. Así, la empresa épica del poema Milton se librará en los confines de la mente.

William Blake establece en el prefacio en verso de Milton que su guerra atañe al ámbito de la psique: "I will not cease from Mental Fight". ${ }^{3}$ Como argumentaré en este ensayo, Milton a Poem constituye una operación poética de suma introspección psicológica. William Blake lucha en este poema consigo mismo. El poema es más que una interesante composición poética: es casi una suerte de psicoanálisis con recursos mitopoéticos. El estilo de Milton participa del mismo lenguaje que imbuye a la expresión de la mente y allí radica, al menos en parte, la dificultad del poema. Este tipo de lenguaje mental no discrimina racionalmente entre distintos tipos de símbolos y referentes; al igual que con los sueños, todo puede convertirse en parte de la articulación psicológica y la significación de cada elemento puede ser al mismo tiempo concreta y múltiple.

En gran medida, Blake recurre a una tradición muy fuerte que caracteriza al poeta como profeta satírico y en ello Blake logra hacerse de los "más sublimes trofeos de la guerra mental" (Tannenbaum, 1976: 77). Este recurso no nace en Milton a Poem sino en The Marriage of Heaven and Hell. Ya allí las categorías de Infierno y Cielo se entienden no como sitios geográficos sino como regiones de la mente (ibid.: 87), al igual que asevera el Maligno en Paradise Lost. Es justamente este carácter mental de ambas sagas lo que deriva en la aparente falta de coherencia narrativa de Milton a Poem, pues no se trata de un poema narrativo en el sentido tradicional, sino de la

\footnotetext{
${ }^{3}$ Pensar de igual modo en The Mental Traveller, obra donde Blake también desarrolla una profunda disertación al mismo tiempo introspectiva y subversiva.
} 
narración poética de sucesos simultáneos en diferentes planos de existencia — la vigilia y el ensueño, el mundo físico y el espiritual.

A diferencia de otros textos épicos — con los cuales Blake compara su Milton—, este poema no abunda en interminables y encarnizadas batallas, sino que se circunscribe en gran medida en una lucha mental. Las verdaderas batallas no son corporales, sino espirituales. Se trata de un presupuesto que ya Blake había asentado en las dos últimas estrofas del prefacio:

Bring me my Bow of burning gold;

Bring me my Arrows of Desire;

Bring me my Spear; O clouds unfold!

Bring me my Chariot of fire!

I will not cease from Mental Fight,

Nor shall my Sword sleep in my hand,

Till we have built Jerusalem

In England's green \& pleasant Land.

De algún modo, Blake emula las palabras de Satán en Paradise Lost en un par de pasajes:

The mind is its own place, and itself

Can make a heaven of hell, a hell of heaven (ParL I. 254-55)

y:

Which way I fly is Hell; myself am Hell (ParL IV.75).

Milton, a su vez, retoma la tribulación inherente de Mefistófeles en Dr. Faustus, de Christopher Marlowe:

\section{FAUSTUS Where are you damned?}

MEPHISTOPHILIS In hell.

FAUSTUS How comes it then that thou art out of hell?

MEPHIST Why, this is hell, nor am I out of it (DrF III.73-76).

Y más adelante:

FAUSTUS $\quad[. .$.$] Tell me, where is the place that men call hell?$

MEPHIST Under the heavens.

FAUSTUS Ay, so are all things else; but whereabouts?

MEPHIST Within the bowels of these elements, Where we are tortured and remain for ever. Hell hath no limits, nor is circumscribed 


\section{$68 \square$ THE DEVIL'S PARTY: MILTON EN LA POÉTICA}

In one self place, but where we are is hell,

And where hell is, there must we ever be.

[...] All places shall be hell that is not heaven (DrF V.115-25).

En los tres autores existe una tendencia importante a caracterizar el infierno y el cielo como estados mentales más que como lugares más o menos topológicos. De hecho, todos los acontecimientos narrados en Milton a Poem suceden en un instante del tiempo secular, y ese voluptuoso instante responde a la visión profética de Blake. En palabras de Bloom, "La cuestión principal en Milton es si Blake puede seguir a Milton en el desierto de la profecía, y si Milton (al darle a Blake coraje visionario) puede eliminar la última parte de su propia historia que todavía pertenece a Satán” (Bloom, 1999: 202). Nos queda a nosotros aventurar una conclusión a esta interrogante.

Uno de los puntos de partida de Milton se presenta justo antes del prefacio en verso, donde en un fragmento en prosa Blake acusa los errores estéticos del periodo clásico, en contraposición con la superioridad artística de la Biblia; dice, además, que los autores William Shakespeare y John Milton habían sucumbido a esta perniciosa influencia: "Shakspear \& Milton were both curbd by the general malady \& infection from the silly Greek \& Latin slaves of the Sword" (Mil, pref.). La referencia negativa a la literatura clásica está también dirigida a los poetas neoclásicos.

El poema, escrito en versos de catorce sílabas, se divide en dos Libros. El primero, semejando el mismo recurso lírico de Milton en su Paradise Lost, comienza por situar el contexto. La pesadumbre de Milton se pone de relieve; el poeta providencial se encuentra infeliz aunque reside en el cielo (Mil I: 1.18/ E p. 96), ${ }^{4}$ en buena medida porque no ha sabido hacer las paces con sus tensiones sensuales: "in soft delusions / Of varied beauty, to delight the wanderer and repose / His burning thirst \& freezing hunger!..." (Mil I.: 1.3-5/E p. 96). Por esa razón, su Emanación Séxtuple vaga penitentemente en la eternidad (Mil I: 1.19-20/E p. 96). Tal prurito lo ha dejado insatisfecho, con ansias de saciar su sed y hambre sensorial.

Blake procede a invocar a las musas (Mil I: 1.1 ss./E p. 96), lo que curiosamente deriva del uso clásico de Homero y Virgilio a pesar del ataque expuesto en el prefacio:

Say first! What mov'd Milton, who walked about in Eternity

One hundred years, pondring the intricate mazes of Providence

Unhappy tho in heav'n... (1.16-18/E p. 96).

La invocación de Blake deriva directamente de Paradise Lost:

\footnotetext{
${ }^{4}$ Los números romanos se refieren al Libro correspondiente de Milton: A Poem in Two Books; el primer número arábigo corresponde con la lámina y el segundo con el número de verso, según la versión de Essick y Viscomi (Blake, 1998). La secuencia de láminas siempre ha sido un punto de desacuerdo entre los estudiosos de Blake, así que doy también el número de página en la edición de David Erdman (Blake, 1988).
} 
In thoughts more elevate, and reasoned high

Of Providence, Foreknowledge, Will, and Fate

[...]

And found no end, in wandering mazes lost (ParL 2.599-61).

Y:

...deep on his front engraven

Deliberation sat, and public care,

And princely counsel in his face yet shone

Majestic, though in ruin... (ParL 2.302-305).

Blake apela a las Musas para poder vislumbrar el remordimiento que padece Milton en la eternidad y su subsiguiente decisión de buscar reconciliarse al fin con su Séxtuple Emanación, a saber: sus tres esposas y tres hijas. La trama del poema, sin embargo, deviene más compleja, pues Blake presenta a varios personajes de su invención (¿visión?) además de John Milton. Aun más: en algún punto, las figuras de Los, Blake y Milton llegan a fusionarse, lo que podría apuntar hacia una comunión ideológica y estética entre los tres personajes. Uno de los primeros personajes en hacerse presente es Los.

Tras el breve preludio, Milton a Poem comienza de lleno con el fragmento conocido como la "Canción del Bardo" (Mil I: 1.24 ss./E p. 96 ss.), que no ofrece una narración cronológica de eventos, sino que más bien introduce la pugna entre Palamabron y Satán. Esta Canción comprende alrededor de once láminas del poema. ${ }^{5}$ La Canción del Bardo elabora sobre la revuelta de Satán y sobre la creación del infierno, el pecado y la muerte, además de algunos errores religiosos, particularmente del calvinismo; tras refutarlos, ofrece una tipología de los seres humanos. Además, Satán, pensando que hace lo correcto, pide a Los realizar los trabajos de Palamabron en bienestar de éste; ello, sin embargo, deriva en un verdadero desastre que duplica las tensiones y remordimientos también experimentados por Milton. Así, Rintrah, baluarte de la ira, se ve forzado a intervenir.

Durante este mismo pasaje encontramos una categorización ontológica de los seres humanos, la cual corresponde con los personajes recién citados, a saber: Satán, Palamabron y Rintrah. La humanidad, según esta concepción, se divide en tres clases: los Elegidos, los Redimidos y los Réprobos (Mil I: 1.26, 3, 4.35-7, 5, 6.36-7 ss., 24.31-37/E pp. 96-103, 122). Según Blake, los Réprobos constituyen los verdaderos creyentes, mientras que los Elegidos perpetúan el verdadero mal, pues están inmersos en una moral narcisista. Es a ojos de los Elegidos que los verdaderos creyentes aparecen como Réprobos, una tergiversación que recuerda las técnicas narrativas empleadas en The Marriage of Heaven and Hell, donde el axioma "Good is Heaven [...]

\footnotetext{
${ }^{5}$ En su totalidad, Milton a Poem comprende unas cuarenta y cinco láminas, según la copia utilizada. La versión en Blake 1998 consta de cuarenta y seis láminas; la de Blake 1988 señala versiones de cuarenta y tres y de cincuenta láminas.
} 
Evil is Hell" adopta tintes sarcásticos a lo largo del texto. Es importante advertir que las tres clases de hombres son producto de la Imaginación y, por ende, hijos de Los.

Si expandimos esta tipología, las correspondencias revelan los distintos niveles que Blake maneja simultáneamente:

Los fuertes-Réprobos-Rintrah

Los bellos-Redimidos-Palamabron

Los espantosos_Elegidos_-Satán

Según la cosmología blakeana, Albión, el Hombre Universal, padece una caída del estado divino y pleno, dividiéndose en tres clases de hombres: los fuertes, los bellos y los espantosos. La primera clase se refiere a los iconoclastas e iracundos, cuyo mejor exponente es el profeta Elías, más tarde manifestado en Juan Bautista. La tercera clase está conformada por los agentes de la moral estéril, aquellos que velan por la Ley y las convenciones religiosas y sociales. La segunda clase condensa a los hombres honestos, gente más cautelosa y mesurada que oscila entre las otras dos clases ( $c f$. Frye, 1990: 332-334). A veces, sin embargo, un individuo puede mutar de clase, pues no se trata de naturalezas del todo inherentes, sino de estados susceptibles de ser transformados.

Después de que la voz poética describe a estas clases de hombres, John Milton, residente en un plano celestial indefinido en la eternidad, decide regresar para purgar sus errores. Decide, pues, abandonar sus ideales puritanos e ingresar a la "Muerte Eterna" (Eternal death), toda vez que encarnar como hombre en la tierra supone el inexorable destino de morir (Mil I: 14/E p. 109). Milton está ya consciente de que sus desviaciones lo han hecho perpetuar ideas erróneas: "I in my Selfhood am that Satan" (Mil I: 14.30). Milton, pues, buscará la autoaniquilación o, mejor dicho, la aniquilación de una porción de su ser, a saber: su propia e injusta representación de Satán. Antes de eso, el poema ha estado en voz del Bardo, quien antes de la decisión de Milton de regresar a la tierra se funde con el fallecido poeta (Mil I: 14.9/E p. 109). Milton escucha el Canto del Bardo desde la eternidad y ello lo impele a corregir sus errores, lo cual también implica redimir a Ololon, su Emanación femenina y séxtuple. Sin esta fusión, Milton el personaje no podría reunir el temple necesario para encarnar y disipar sus errores ideológicos anteriores. Si ha de descender a la tierra, ello será sólo una vez que esté inspirado y animado por el Genio Poético, que en este poema adopta, entre otras manifestaciones, la forma de la Voz del Bardo.

Aunque ha descendido a la tierra bajo la forma de un cometa y ha entrado en el pie izquierdo de Blake (Mil I: 14.47-50 / E p. 110), Milton no constituye una pieza narrativa central en el primer Libro. Como mencioné ya, un gran número de versos del poema se centran en la Canción del Bardo y las pugnas entre Palamabron, Rintrah y Satán. Además, una gran parte del Libro I de Milton "describe el mundo de los tiempos de Blake, que está demasiado maduro para el apocalipsis... La función de Milton-Blake-Los es adquirir una visión de este mundo que sea el contrario trascendente de la visión de éste de sí mismo" (Bloom, 1999: 208). De este modo, Blake es guiado por Los y entra en 
Golgonooza, la Ciudad del Arte, bajo los auspicios del espíritu de la creatividad profética. Pero en el trayecto, la voz poética también se dedica a describir diversos órdenes y constituciones de las realidades fenoménicas y nouménicas. Un pasaje en particular es aquel donde se definen dos límites a la realidad divina: la Opacidad y la Contracción (Mil I: 13.20-21). La Opacidad adquiere la forma de Satanás y la Contracción, la de Adán. La tensión que existe entre ambas entidades imbuye el pensamiento blakeano y está articulada de diversos modos a lo largo de su obra.

Es importante advertir que la pugna entre Palamabron, Los y Satán contenida en la Canción del Bardo emula de una manera compleja y alegórica una situación particular que Blake experimentó durante su estadía en Felpham. William Hayley, un reputado patrono de las artes en esa época, había asumido el mecenazgo del artista Blake, pero lejos de dejarlo producir libremente, le encomendó una serie de obras más fáciles de acoplar con la sensibilidad convencional y social en turno. Las "extravagancias" de Blake, se entiende, estaban vedadas. Las ambiguas actitudes de Blake para con su patrono (Satán, en la Canción del Bardo) se reflejan en Palamabron y Rintrah, respectivamente el espíritu manso y el espíritu impulsivo. Si Hayley fue "amigo corpóreo y enemigo espiritual" de Blake, acaso Milton fue su contrincante poético/ideológico, mas aliado espiritual.

Milton se ha fusionado primero con la Voz del Bardo y después ha entrado en Blake. Aún más: Los, personaje blakeano que representa la imaginación y la facultad creativa, se fusiona con Blake (Mil I: 21.4-14/E pp. 116-117). La relación ontológico-estética se ha completado; ahora los dos poetas, vinculados por el Genio Poético, pueden emprender juntos el viaje. Milton ha tenido que dictar a sus esposas e hijas sus visiones y sus nuevas percepciones (Mil I: 16.9 ss./E p. 110), desde luego una sátira blakeana de los tiempos en que, ciego e incapaz de escribir por sí mismo, Milton tuvo que dictar sus composiciones.

Un paso forzado en la empresa reivindicatoria de Milton es enfrentarse con Urizen, la encarnación de los tiránicos poderes de la Razón en el imaginario blakeano. Ambos se enfrentan a orillas del río Jordán tras guardar unos minutos de silencio. Urizen toma con su "fría mano" un poco de agua del río y la vierte encima de la cabeza de Milton. En respuesta, Milton toma arcilla roja de Succoth, una ciudad al este del Jordán, y la moldea con sus manos, "rellenando los surcos de muchos años", desde los pies de Urizen, de modo que reviste a Urizen con un ropaje nuevo (Mil I: 18.6-14/E p. 112). Lo convierte, pues, en una especie de Nuevo Adán. Si Urizen pretendía bautizar a Milton con un frío y estéril intelectualismo, Milton hace de su contrincante un Nuevo Hombre, acompañado de cosecha y florecimiento. Al mismo tiempo, es posible que este enfrentamiento parodie al bíblico Jacob (Bloom, 1999: 205; cf. Génesis: 32, y Oseas: 12).

El Libro II de Milton comienza con las descripciones y percepciones de Beulah, la primera fase a la que desciende la Emanación femenina de Milton (Ololon), y de Ulro, el universo newtoniano de la Razón y el materialismo. Después, Ololon se aparece ante Blake, en su jardín de Felpham (Mil II: 36 y 37/E pp. 136-137); aunque Blake la invita a hospedarse con él y su esposa, Ololon replica que anda en busca de Milton. De 


\section{$72 \square$ THE DEVIL'S PARTY: MILTON EN LA POÉTICA}

inmediato, en el mismo Jardín se aparece la Sombra de Milton. Ambos sostienen un intenso diálogo, en el cual Ololon reconoce que ella ha sido la causante del Deísmo y de la Religión Natural. Por su parte, Milton explica la batalla que ha de librarse con su negación y sus Contrarios: a la Negación (Satán) hay que aniquilarla; a los Contrarios (la Emanación séxtuple) hay que conciliarlos (II: 42.4-22/E pp. 141-142). El clímax del parlamento de Milton es el siguiente:

All than can be annihilated must be annihilated

That the Children of Jerusalem may be saved from slavery

There is a Negation, \& there is a Contrary

The Negation must be destroyd to redeem the Contraries

The Negation is the Spectre; the Reasoning power in Man

This is a false Body: an Incrustration over my Immortal

Spirit; a Selfhood, which must be put off $\&$ annihilated alway

To cleanse the Face of my Spirit by Self-examination.

El dios de Paradise Lost, identificado por Blake con Urizen y la razón tiránica, debe ser eliminado para permitir la emancipación de los hijos de Jerusalén (los hijos, también, del gigante Albión). Poco a poco, los velos que evitan la identificación plena entre Milton y Ololon se disipan y pueden entonces emerger como Un Solo Hombre, parangón de una humanidad resucitada.

Si bien es cierto que Blake consideraba que Milton había albergado perspectivas erróneas en términos de política y teología, seguía considerándolo como un verdadero poeta y por ello constituía un modelo digno de tomar en cuenta. Una de las características que Blake retoma de la poética de Milton es el trastrueco de las convenciones sobre la épica: “...Milton and Blake's epics rely upon satire to subvert the epic tradition, 'knocking away its usual underpinnings, the conventions the form accumulated, in order to create epic anew" (Tannenbaum, 1976: 94). Se trata, pues, de revitalizar la forma épica, pero centrándola en un contexto cristiano y teológico, más que meramente bélico. Ello no implica la pérdida del sentido heroico; sólo lo recontextualiza. Al mismo tiempo, Blake también renueva los alcances de su precursor: "...he corrects and interprets Milton's vision by redefining his predecessor's own theory and practice" (idem). Lo que Milton considera el Mal — a ojos de Blake - no es sino una fuerza que se rebela ante un dios despótico y autoritario; para Blake, el verdadero Mal son las fuerzas opresoras que coartan las libertades individuales.

Pero es importante advertir que si Blake considera que Milton puede obtener la redención, a pesar de sus concepciones maniqueas y estériles, es porque a través de su poesía se revelaba, aunque fuese de manera inconsciente, una gran red de tensiones binarias ( $c f$. Nuttall, 2007: 86-87). Blake habría de considerar que estas pulsiones, por llamarles de algún modo, no eran sino destellos del Genio Poético inherente en la mente de Milton el poeta. Es gracias a estas tensiones que - como advirtieron Blake y otros poetas románticos - los versos de mejor manufactura en Paradise Lost, los de mayores ímpetus, están entonados por voces demoniacas, no angélicas: 
To reign is worth ambition, though in hell:

Better to reign in hell than serve in heaven (ParL I. 262-263).

Un verso como éste no puede sino azuzar los sentimientos que buscan la libertad moral, social y artística, un sentimiento que Blake abanderó a lo largo de su vida y obra. Para ello es vital redefinir los valores de Bien y Mal, Cielo e Infierno, Ángel y Demonio.

Resulta igualmente significativa la descripción de la penetración de Milton en Blake:

Then first I saw him in the Zenith as a falling star,

Descending perpendicular, swift as the swallow or swift;

And on my left foot falling on the tarsus, enterd there (Mil I: 14.47-49 / E p. 110).

La imagen y la descripción están basadas en un pasaje importante de Paradise Lost. Allí, encontramos que la caída de Mulciber es el antecedente de la caída de Milton en el poema de Blake:

Men called him Mulciber, and how he fell

From Heaven they fabled, thrown by angry Jove

Sheer o'er the crystal battlements: from morn

To noon he fell, from noon to dewy eve,

A summer's day, and with the setting sun

Dropped from the zenith, like a falling star,

On Lemnos, the Aegaean isle (ParL I.740-46; mi énfasis).

Recalco que en Milton a Poem la caída y penetración del espíritu de Milton en el pie izquierdo de Blake (como un cometa) copia el descenso precipitado de Mulciber-Lucifer en Paradise Lost. Por tanto, podemos deducir que en el poema de Blake se trata de un John Milton de algún modo empañado, obnubilado y confundido con el antagonista de su obra. Pero, al mismo tiempo, resulta más que pertinente que sea justamente emulando a un demonio (estandarte de la energía blakeana) como tenga que descender.

Blake consideraba que Milton, en realidad, pertenecía al Bando de los Demonios (la Imaginación y la Energía), pero sus ideas conservadoras le impedían asumirlo abiertamente. De manera similar, Blake, bajo el patronazgo de William Hayley, corrió también el riesgo de ver mermadas sus facultades creativas. Por ello, ambos tienen que emprender un intenso viaje intelectual e imaginativo en el poema Milton para desencadenar sus impulsos, para desembarazarse de los grilletes de la razón y el orden en sus formas tiránicas y al mismo tiempo seductoras.

Milton a Poem es en realidad menos un texto sobre John Milton que sobre William Blake. Milton, pese a ser un catalizador del poema (en sentido externo e interno), funciona más como un símbolo conductor para enmarcar la pugna psíquica de Blake. Lo que Blake hace en Milton a Poem es en realidad vindicarse a sí mismo, lidiar con sus propias tensiones creativas. Para ello, Blake enmarca esta ansiedad en el lenguaje 


\section{THE DEVIL'S PARTY: MILTON EN LA POÉTICA}

visionario que ya había estado desarrollando en obras anteriores. Se trata de sus propias ansiedades, pero también de tensiones inherentes al ser humano en un sentido amplio. En un punto crucial del poema, de hecho, Milton, Blake y Los también se combinan. Una vez que Milton ha penetrado en el pie de Blake y que éste se ha atado las sandalias para transitar por el mundo natural, el Profeta Los se acerca a Blake y se funde con el poeta (Mil I: 21.4-14 /E p. 116). Puesto que la batalla épica de Milton corresponde a un ámbito psíquico y no a uno marcial, todos los acontecimientos son percibidos merced a una suerte de trance visionario. Los ejércitos que combaten en el poema de Blake no están conformados por soldados y armamento, sino por visiones, tanto en el sentido de "puntos de vista particulares", como en el de "imágenes percibidas por la representación imaginativa".

Aquí me gustaría resaltar un punto crucial en la experiencia visionaria que supone el poema Milton. Aun más que hablar de una contemplación visual/imaginativa, deberíamos entender el contenido y la forma de Milton como una suerte de segmentación psíquica. Si leemos con atención el poema, habremos de darnos cuenta de que, en estricto sentido, Blake no ve a John Milton hacer y decir: es él mismo (William Blake) quien ejecuta las acciones épicas; Milton las realiza a través de él (es justamente Blake quien percibe a Ololon en primer lugar). Recordemos también que en primera instancia Milton se le introdujo a Blake por el talón izquierdo: no se le apareció enfrente, ni lo soñó, ni lo escuchó desde el más allá. En otras palabras: en el poema no hay un Blake más un Milton, sino un Milton en Blake.

La segunda vez que Blake se refiere a la inserción de Milton en su pie, expresa lo siguiente:

But Milton entering my Foot; I saw in the nether

Regions of the Imagination; [...]

But I knew not that it was Milton, for man cannot know

What passes in his members till periods of Space \& Time

Reveal the secrets of Eternity [...] (Mil I: 20.4-10 / E p. 115).

Este pasaje parece apuntar justamente hacia esa confusión de realidades psíquicas en la persona de William. La alternancia de "mentalidades" o aun de "personalidades" se refuerza con la instrucción que los Siete Ángeles imparten a Milton: "We are not Individuals but States: Combinations of Individuals” (Mil II: 32.10 / E p. 131). En este sentido, y según la lógica interna del poema, Palamabron, Rintrah, Milton, Satán y aun Albión no son sino "estados" de la existencia de William Blake, porciones de su propio ser.

Para que Blake pudiera lidiar con las ansiedades que ocasionaba su relación con Hayley - y posiblemente otras —, hubo de "invocar" al espíritu de Milton (él mismo sujeto a convenciones y restricciones). Juntos habrían de buscar la aniquilación de sus fardos y la consecución de la redención. Verdaderamente, Milton a Poem es una suerte de experiencia casi esquizofrénica, donde Blake adopta varias manifestaciones o, mejor 
dicho, donde varios personajes operan en niveles distintos; uno de éstos es justamente el de la representación de pulsiones distintas de la psique blakeana. Recapitulando: el Bardo se funde en Milton, que se funde en Blake, en quien se funde también Los y quien(es) eventualmente emerge(n) como Albión. Recordemos también que en la lectura que he favorecido en este trabajo Palamabron y Rintrah constituyen dos binomios de la mente de Blake. No hay que olvidar tampoco que la clasificación de los hombres en Elegidos, Redimidos y Réprobos no constituye una tipología absoluta, sino que representa varios estados susceptibles de ser transformados - pero de preferencia transmutados vía el fuego "infernal” de la Imaginación.

William Blake retoma no sólo la poética miltoniana sino a la figura misma de John Milton como un antecesor y símbolo ineluctable. Al buscar la reivindicación del "empañado" Milton, Blake realiza una lectura muy interesante y profunda. A lo largo de esta suerte de redención (que es también una redención propia), Blake también redefine el cristianismo. Curiosamente, al encauzar a Milton por el sendero de la reconciliación de contrarios, Blake no retorna a un cristianismo más puro, sino que forja un nuevo mapa conceptual. En ese sentido, los motivos y personajes bíblicos operan como símbolos de la poética blakeana al igual que las figuras de su propia concepción. Todos estos símbolos conducen no a la obtención del cielo cristiano y la gracia divina, sino a un proceso de autorrealización, un complejo procedimiento de individuación extrema en el cual el individuo debe conciliarse con las fuerzas antagónicas de su psique.

En un momento, hacia el final del poema, Blake regresa a su estado mortal: sale de su trance visionario, en el cual percibió el retorno y las empresas de John Milton (Mil II: 45: 25-35/E pp. 143-144). En ese mismo trance, como hemos visto, Blake de algún modo estuvo poseso por el espíritu de Milton. Una vez que Milton logra disipar sus concepciones teológicas erradas es capaz de reunirse con Ololon, su Emanación Femenina, o sea los espíritus de sus esposas e hijas en la Eternidad. Jesús se yergue, glorioso y satisfecho con la conciliación de Ololon. Así, Albión el Hombre Universal, del cual todos los seres vivos participamos, está preparado para despertar completamente y presenciar el Día del Juicio y para reunirse con su propia emanación: Jerusalén. William Blake, ya de vuelta en el plano mundano en Felpham, ve junto con su esposa Catherine ("my sweet Shadow of Delight") el vuelo de la alondra, cuyo canto se une a los rugidos de Los para anunciar la proximidad de la cosecha y la vendimia del Día del Juicio Final, prefigurado en el Libro I (I: 24 / E pp. 121-122; $c f$. Apocalipsis 14: 14-20). Pero ésa es otra historia, una que continuará en el libro Jerusalem: the Emanation of the Giant Albion.

\section{Obras citadas}

BLAKE, William. 1998 (1993). Milton a Poem. Ed., introd. y notas Robert N. EssicK y Joseph VISCOMI. Nueva Jersey: The William Blake Trust / Princeton University Press. (The Illuminated Books, 5) 
. 1993. Selected Poems. Ed. Peter BuTTER. Londres / Vermont: Everyman. . 1988. The Complete Poetry and Prose of William Blake. Ed. David ERDMAN. Comentario Harold Bloom. Nueva York / Londres / Toronto / Sydney: Anchor Books / Doubleday.

Bloom, Harold. 1999. La compañía visionaria: William Blake. Trad. Mariano Antolin RATTO y Pablo GiAnerA. Buenos Aires: Adriana Hidalgo Editora.

DAMON, Foster. 1988 (1965). A Blake Dictionary. The Ideas and Symbols of William Blake. Con un nuevo prefacio de Morries EAVES. Hanover / Londres: University of New England Press.

FRYE, Northrop. 1990 (1947). Fearful Symmetry. A Study of William Blake. Princeton University Press.

Marlowe, Christopher. 1973. Dr. Faustus. En The Oxford Anthology of English Literature, vol. I. Nueva York / Londres / Toronto: OUP. Pp. 848-899.

Milton, John. 1996 (1674). Paradise Lost. Hardmonsworth: Penguin.

NutTall, A. D. 2007 (1998). The Alternative Trinity. Gnostic Heresy in Marlowe, Milton, and Blake. Oxford / Nueva York: Oxford University Press.

ScHOCK, Peter A. 1993. "The Marriage of Heaven and Hell: Blake's Myth of Satan and Its Cultural Matrix”. ELH, vol. 60, núm. 2. Pp. 441-470.

SCHUCARD, Marsha Keith. 2006. Why Mrs Blake Cried. William Blake and the Sexual Basis of Spiritual Vision. Londres: Century.

SERRA, Cristóbal. 1992. Pequeño diccionario de William Blake. (Caracteres simbólicos.) Barcelona: Alejandría.

TAnnEnBAum, Leslie. 1976. "Blake's News From Hell: The Marriage of Heaven and Hell and the Lucianic Tradition”. ELH, vol. 43: 1. Pp.74-99. 